\title{
Human Enhancement: Einführung und Definition
}

Nikola Biller-Andorno, Michelle Salathé

Korrespondenz:

Schweizerische Akademie der Medizinischen Wissenschaften (SAMW)

Petersplatz 13

CH-4051 Basel

mail[at]samw.ch
Unter dem Stichwort «Human Enhancement» werden medizinische Interventionen diskutiert, die sich nicht auf die Therapie von Krankheiten, sondern auf eine Verbesserung nichtpathologischer Merkmale richten. Dabei werden zahlreiche Fragen aufgeworfen: Wo verläuft eigentlich genau die Grenze zwischen Therapie und «Enhancement»? Ist «Enhancement» moralisch verwerflich oder vielmehr ein Teil unseres Alltags, auch des medizinischen? Führt «Enhancement» dazu, dass diejenigen, die schon jetzt privilegiert sind, noch «schöner und reicher» werden, während andere immer mehr den Anschluss an gesellschaftliche Chancen und Erwartungen verlieren?

Der vorliegende Bericht wendet sich diesen gesellschaftlichen und ethischen Fragen zu. Dabei fokussiert er besonders auf die Rolle der Ärzteschaft. Sie sind die Berufsgruppe, die eine wichtige Rolle in der Beschaffung, aber auch in der Bewertung von Enhancementprodukten spielen würde, durch Beiträge zur Grundlagen- und klinischen Forschung, durch Beratungsgespräche mit Patienten und auch durch die Entwicklung berufsethischer Standards. Viele Wirkstoffe, die als Enhancer verwendet werden können, sind verschreibungspflichtig, und so werden Ärztinnen und Ärzte ganz unmittelbar mit der Frage konfrontiert, ob sie solche Produkte zugänglich machen sollen oder nicht. Dabei gilt es, zwei Ebenen zu berücksichtigen: Zum einen geht es um den individuellen Patienten - seine Präferenzen, seinen Nutzen, sein Risiko. Zum anderen geht es um gesellschaftliche Auswirkungen des Einsatzes von Medikamenten zur Leistungssteigerung bei Gesunden.

Zur Komplexität der Debatte trägt auch bei, dass Human Enhancement ein sehr heterogenes Phänomen ist, das zudem verschiedenste Bereiche der Medizin tangiert, z. B. Reproduktionsmedizin, Neurowissenschaften, Plastische Chirurgie oder Ernährungsmedizin. Eingriffe unterscheiden sich u. a. mit Blick auf ihre Invasivität, Wirkungsweise, Effekte, Risiken und Nebenwirkungen sowie deren Dauer und Reversibilität. So umfasst der Begriff «Enhancement» gleichermassen die Einnahme von Vitaminpillen mit vorübergehenden Effekten und geringem Risiko wie das Einpflanzen eines Neurochips mit Auswirkungen auf die Gehirnfunktion des betreffenden Individuums oder die genetische Modifikation der Keimbahn, die in nachfolgenden Generationen fortwirkt [1a].

Der vorliegende Bericht fokussiert weniger auf spekulative, zukunftsgerichtete Szenarien, die eine
«Human Enhancement» bezeichnet medizinische oder biotechnologische Interventionen, deren Zielsetzung nicht primär therapeutischer oder präventiver Art, sondern eine «Verbesserung» nichtpathologischer Merkmale ist. Eine Arbeitsgruppe der Akademien der Medizinischen Wissenschaften (SAMW) und der Geistesund Sozialwissenschaften (SAGW) hat die ethischen Fragen des Enhancements vertieft untersucht und ihre Analysen und Empfehlungen in der Broschüre «Medizin für Gesunde?» (www. akademien-schweiz.ch $\rightarrow$ Projekte und Themen) veröffentlicht. In loser Folge erscheinen in der SÄZ Auszüge aus dem Bericht.

«Verbesserung» der menschlichen Spezies in den Blick nehmen, sondern auf eine graduelle Verschiebung der Leistungsgrenze mit heute oder in absehbarer Zeit verfügbaren Mitteln. Was das pharmakologische Enhancement betrifft, so gibt es bereits heute ein breites Spektrum an Substanzen, die teils den Drogen und Dopingmitteln (z.B. Kokain), teils den Arzneimitteln (z.B. Methylphenidat) und teils Lebens- und Genussmitteln (z. B. Energydrinks) oder Kosmetika (z. B. Anti-Aging-Cremes) zuzuordnen sind [1b]. Manche Enhancer, wie Koffein oder Alkohol, kommen in unserer Gesellschaft schon lange zum Einsatz, während andere Substanzen (z. B. Modafinil) erst in letzter Zeit verstärkt «off-label», also jenseits des zugelassenen Gebrauchs für bestimmte Erkrankungen, eingesetzt werden.

In der Medizinethik wird die Diskussion um das Enhancement bereits seit Jahrzehnten geführt, etwa zu Bereichen wie der Keimbahntherapie, der kosmetischen Chirurgie oder dem Anti-Aging. In den letzten Jahren hat sich auch eine Reihe von politik beratenden Institutionen der Thematik zugewandt,

\author{
Mitglieder der Arbeitsgruppe \\ Prof. Dr. med. Dr. phil. Nikola Biller-Andorno (Vorsitz) \\ Prof. Dr. med. Anne-Françoise Allaz \\ Dr. phil. Gaia Barazzetti (bis 2010) \\ lic. ès lettres Nadja Birbaumer, SAGW \\ PhD Susanne Brauer, NEK-CNE \\ Prof. Dr. med. Jürg Kesselring \\ Prof. Dr. med. lic. phil. Iris Ritzmann \\ Dr. phil. Simone Romagnoli \\ Dr. biol. Adrian Rüegsegger, TA-SWISS \\ lic. iur. Michelle Salathé, MAE, SAMW \\ PD Dr. theol. Markus Zimmermann-Acklin
}


darunter der U.S. President's Council [2], das Büro für Technikfolgen-Abschätzung beim Deutschen Bundestag [3], das niederländische Rathenau-Institut [4] und das Science-and-Technology-Options-Assessment-(STOA-)Büro des Europäischen Parlaments [5].

In der Tat wirft Enhancement interessante rechtliche Fragen auf, etwa bezüglich der Reichweite der individuellen Autonomie über den eigenen Körper und der Gründe, die eine gesetzliche oder standesrechtliche Einschränkung rechtfertigen. Als weitere Frage lässt sich beispielhaft anführen, inwieweit medizinisch indizierte Behandlungen von Folgen, die aus der Anwendung von Enhancement resultieren, von der obligatorischen Krankenversicherung übernommen werden sollen. Nachfolgend soll kurz der aktuelle rechtliche Rahmen in der Schweiz skizziert werden. Die Ausführungen stützen sich wesentlich auf das von der Nationalen Ethikkommission (NEK-CNE) in Auftrag gegebene Gutachten [6] (nachfolgend NEK-CNE-Gutachten) und die rechtliche Beurteilung im Rahmen der TA-SWISS-Studie [1c].

Auf Verfassungsebene stehen diverse Grundrechte in einem Bezug zum Enhancement, namentlich die Menschenwürde (Art. 7 BV), das Recht auf Leben und auf persönliche Freiheit (Art. 10 BV) oder der Schutz der Kinder und Jugendlichen (Art. 11 BV). Relevant ist die Frage, inwieweit sich aus der Verfassung Grundrechtsansprüche (z.B. Recht der Konsumenten auf Nutzung von Enhancement, der Forschenden auf Durchführung von Forschungsprojekten zu Enhancement usw.) und Schranken ableiten lassen. Im Bereich der Erlasse auf gesetzlicher Ebene empfiehlt der TA-Bericht eine Unterscheidung nach Handlungsbereichen: Behandlungsverhältnis (insb. Aufklärungs- und Sorgfaltspflichten), Forschung, Berufszulassung, Marktzulassung sowie sozialer Ausgleich. Er setzt sich mit der Frage auseinander, in welchen Bereichen bereits spezifische Regeln für Enhancementbehandlungen bestehen oder ableitbar sind und wo solche fehlen. Zusammenfassend kann festgehalten werden, dass der TA-Bericht durchaus Regelungsbedarf für den Gesetzgeber sieht. So hält er es für wünschbar, dass dieser sich ausdrücklich zum Behandlungszweck des Enhancements äussert und Klarheit darüber schafft, ob Personen, die Enhancement anbieten, einer Berufszulassung bedürfen. Ausserdem müsste eventuell geklärt werden, inwieweit das Heilmittelrecht zur Anwendung kommt und welche Regeln bei der Verschreibung für den Off-Label-Use gelten. Schliesslich stelle sich die Frage, ob es Regelungen in anderen Wettbewerbsbereichen als dem Sport, namentlich in der Bildung und der Arbeit, braucht. Zu diskutieren wäre für die Verfasser auch die aktuell bestehende Verpflichtung zur Übernahme der Kosten durch die obligatorische Krankenversicherung, die durch eine medizinisch-indizierte Behandlung eines Folgeschadens von Enhancementanwendungen entstehen würden.
Das NEK-CNE-Gutachten kommt hingegen zum Schluss, dass keine nennenswerten rechtlichen Lücken bestehen und sich für die unterschiedlichen betroffenen Bereiche (Medizinalberufe, Heilmittel usw.) folgende Grenzziehungen ableiten lassen:

- Jegliche absichtliche genetische Veränderung oder Verbesserung von ungeborenen Kindern ist verboten.

- Genetische Analysen dürfen nicht zu Freizeit zwecken oder im Bereich der Arbeit eingesetzt werden.

- Die Entnahme und Transplantation von Organen mit dem Ziel, die Leistung eines gesunden Körpers zu verbessern, ist verboten.

- Die Möglichkeiten, Forschungsvorhaben zur Entwicklung von neuen Methoden und Produkten zur Verbesserung des Menschen durchzuführen, sind eingeschränkt durch das Erfordernis einer Risiko-Nutzen-Analyse.

- Ärzte und weitere Fachpersonen im Gesundheitsbereich sind in ihrer Aktivität an die Regeln der ärztlichen Berufspflicht gebunden, das heisst, sie können Interventionen und Produkte nicht ohne medizinische Indikation einsetzen.

- Swissmedic darf die Einführung von Medikamenten, die weder der Diagnose noch der Prävention oder Behandlung einer Krankheit, sondern ausschliesslich dem Enhancement dienen, nicht zulassen.

- Das Verschreiben von Medikamenten für den Off-Label-Use ist nur unter strengen Voraussetzungen erlaubt; Interventionen im Bereich des Enhancements fallen nicht a priori darunter.

- Das Arbeitsrecht verpflichtet den Arbeitgeber dazu, die Arbeitsbedingungen den Arbeitnehmern anzupassen (und nicht umgekehrt) und verbietet explizite oder stillschweigende Forderungen nach Enhancements bei den Arbeitnehmern.

- Die ärztlichen Interventionen im Bereich des Enhancements erfüllen die Kriterien für die Übernahme dieser Leistungen im Bereich der Sozialversicherungen, KVG oder UVG nicht.

Aufgrund dieser Analyse empfiehlt das NEK-CNEGutachten, dass die in diesen Bereichen tätigen Personen und die Öffentlichkeit, namentlich die Patienten und Konsumenten, klar über die bestehenden Regelungen und deren Grenzen zu informieren seien.

Eine Schwierigkeit in der aktuellen kontroversen Diskussion zum Regulierungsbedarf auf nationaler wie auf internationaler Ebene ist das Fehlen einer Einigung bezüglich der Definition von Enhancement* Auch weitere zentrale Begriffsbestimmungen wie z.B. «Gesundheit - Krankheit», die der Abgrenzung von Therapie und Enhancement zugrunde liegen, werden je nach Rechtsbereich unterschiedlich verwendet und erschweren damit eine rechtswissenschaftliche Debatte der Gesamtentwicklung [7]. 
Wenn normative Fragen mit Blick auf «Human Enhancement» verhandelt werden sollen, ist eine geeignete Definition eine Voraussetzung. Nicht nur weil sie das Feld der Untersuchung absteckt, sondern auch, weil sie erkenntnistheoretische Prämissen bezüglich der Möglichkeit einer «neutralen», objektiven Grenzziehung zwischen gesund und krank, normal und anormal, Enhancement und Therapie bzw. Prävention widerspiegelt.

Die Arbeitsgruppe ging zunächst von der einschlägigen Definition von Eric Juengst - «Eingriffe, die die menschliche Gestalt oder Leistungsfähigkeit über das Mass hinaus verbessern sollen, das für die Erhaltung oder Wiederherstellung der Gesundheit erforderlich ist» [8] - aus, fand sie jedoch in verschiedener Hinsicht nicht zufriedenstellend.

Die in der Arbeitsgruppe angestellten Überlegungen führten zu folgendem Definitionsvorschlag**:

«Medizinische oder biotechnologische Interventionen, deren Zielsetzung nicht primär therapeutischer oder präventiver Art ist und die darauf abzielen, Menschen in ihren Fähigkeiten oder in ihrer Gestalt in einer Weise zu verändern, die in den jeweiligen soziokulturellen Kontexten als Verbesserung wahrgenommen wird.»

- Medizinische oder biotechnologische Interventionen: Diese können sich in mehrfacher Hinsicht unterscheiden: Sie können u.a. dauerhaft oder zeitweise, reversibel oder nicht reversibel, mehr oder weniger risiko- und nebenwirkungsbehaftet sein. Es geht nicht allein um medikamentöse Massnahmen, doch beschränkt sich die Definition auf solche, die sich im medizinisch-biologischen Bereich bewegen. Soziale Interventionen würden
Debatte zu Fragen des Enhancements bei anderen Spezies ihre Berechtigung.

- in ihren Fähigkeiten oder in ihrer Gestalt: Es geht nicht nur um Fähigkeiten, sondern auch um andere physische Aspekte, die in der betreffenden Gesellschaft positiv konnotiert sind, z.B. Körpergrösse, Körperproportionen, «Schönheit». in einer Weise zu verändern, die in den jeweiligen soziokulturellen Kontexten als eine Verbesserung wahrgenommen wird: Was eine Verbesserung ist, kann nicht allgemein formuliert werden, da Enhancement immer auch den Grad der Passung bzw. des Übertreffens spezifischer gesellschaftlicher Erwartungen und Bewertungen reflektiert. Was als Enhancement wahrgenommen wird, ist also vom jeweiligen soziokulturellen Kontext abhängig; dies soll nicht suggerieren, dass in einer Gesellschaft bestimmte Präferenzen und Wertungen von allen Individuen geteilt würden. Vielmehr ist in diesen Fragen von einer gesellschaftlichen Heterogenität auszugehen, daher die Formulierung «in den jeweiligen soziokulturellen Kontexten» im Plural.

- (eine Verbesserung,) deren Zielsetzung nicht primär therapeutischer oder präventiver Art ist: Wiederum geht es um die Intention; die Tatsache, dass die Motivation vielfach nicht eindeutig dem Enhancement einerseits oder den Bereichen «Therapie» und «Prävention» andererseits zuzuordnen ist (z. B. im Falle von Stimmungsschwankungen oder eines «mild cognitive impairment»), wird durch die Qualifikation «primär» Rechnung getragen.

Die von der Arbeitsgruppe entwickelte Definition von «Enhancement» bezieht sich somit nicht in

\section{Ärzte sind die Berufsgruppe, die eine wichtige Rolle in der Beschaffung, aber auch in der Bewertung von Enhancementprodukten spielen würde.}

\footnotetext{
** Die TA-SWISS-Studie [1] hat sich in einem gemeinsamen Abstimmungsprozess mit Blick auf die Komplementarität zum vorliegenden Bericht diese Arbeitsdefinition zu eigen gemacht.
}

damit nicht unter die Definition «Enhancement» fallen. Gezielte verhaltenstherapeutische Massnahmen könnten hingegen durchaus als medizinische Interventionen verstanden werden. Die Grenze ist allerdings fliessend.

- die darauf abzielen: Es geht um die Intention; über die Wirksamkeit wird zunächst noch keine Aussage gemacht. Damit trägt die Definition dem Umstand Rechnung, dass Interventionen, die heutzutage als Enhancement definiert werden, bislang vielfach in ihrer Wirksamkeit umstritten sind.

- Menschen: Eingrenzung für den Zweck des Arbeitsauftrags auf «Human Enhancement»; sicherlich hat darüber hinaus auch eine tierethische deskriptiv-objektivistischer, naturalistischer Weise auf die menschliche Natur bzw. auf objektiv bestimmbare Begriffe von «Gesundheit», «Krankheit» und «Normalität». Die Arbeitsgruppe ist nicht der Ansicht, man könne unabhängig von soziokulturellen Kontexten bestimmen, was als Verbesserung oder gar als relevante Verbesserung zu bewerten sei. Enhancement als realitäts- und alltagsnahes Phänomen (unbeachtet der Transhumanismusdebatte) bezieht sich in der Regel nicht auf Funktionen oder Charakteristika, bei denen eine kulturunabhängige Definition von gesund und krank bzw. normal und anomal möglich wäre. So wird z.B. das Körpergewicht in ästhetischer Hinsicht historisch und kulturell sehr unterschiedlich bewertet. Auch welche Eigen- 
schaften relevant sind, mag von Zeit zu Zeit und von Gesellschaft zu Gesellschaft variieren. Während heute bei uns Jugendlichkeit, Dynamik, Wettbewerbsfähigkeit, körperliche und vor allem kognitive Leistungsfähigkeit betont werden, muss dies nicht für andere Gesellschaften gelten. Insofern kann eine Analyse der «Enhancement»-Angebote bzw. -Versuche aufzeigen, welche Prioritäten und Schwerpunkte die jeweiligen Gesellschaften setzen. Die Definition macht sich somit eine subjektivistischkulturalistische Perspektive zu eigen: Enhancement ist das, was die jeweiligen Gesellschaften als solches erachten.

Enhancement ist in diesem Sinne auch kein neues Phänomen, das durch Biotechnologie ermöglicht worden wäre, sondern ein menschliches Bestreben, mithalten oder besser sein zu können. Enhancement als solches ist nicht historisch einzigartig, sondern Ausdruck dessen, was die jeweilige Gesellschaft als erwünschte und wichtige Merkmale erachtet und was den Betreffenden somit einen Wettbewerbsvorteil bringt, wenngleich entsprechende Anliegen erst in der Moderne an die Medizin herangetragen wurden. Auch wenn Enhancement medizinisch gesehen als irrelevanter «Luxus» betrachtet werden kann, mögen entsprechende Interventionen in bestimmten sozialen Kontexten als dringendes Desiderat erachtet werden, weil dem betreffenden Merkmal dort so hohe Relevanz zukommt - wie z.B. der Sauerstoffaufnahmefähigkeit des Bluts bei Radrennen oder der Fähigkeit, mit wenig Schlaf zu «funktionieren», bei Piloten oder Soldaten. Je nach Umfeld können Enhancementinterventionen also zwischen «überflüssiger Spielerei» und «überlebenswichtig» variieren.

Die Definition bietet keine scharfe Abgrenzung zu Therapie bzw. Prävention, und die Arbeitsgruppe geht auch nicht davon aus, dass eine solche scharfe Abgrenzung hinsichtlich alltagsrelevanter Interventionen möglich ist. Die Interventionen, die an dieser Stelle vor allem in den Blick genommen werden sollen, befinden sich im Grenzbereich dessen, was bei uns heute als «normal» oder "gesund» wahrgenommen wird: Psychopharmaka bei Verstimmungen, die das «Funktionieren» der Betreffenden im Alltag beeinträchtigen, aber nicht notwendigerweise als Depression zu diagnostizieren sind, oder die helfen sollen, eine (allzu?) grosse Schüchternheit in sozialen Kontakten zu überwinden.

Die Definition zielt zudem darauf ab, diejenigen Eingriffe in den Fokus zu nehmen, die derzeit in ethischer Hinsicht diskutiert werden, also gezielte medizinische und biotechnologische Interventionen und weniger alltägliche Interventionen oder unspezifischere Massnahmen wie z.B. die Erziehung von Kindern. Mit dieser Eingrenzung geht jedoch keine moralische Bewertung von medizinisch-biologischen Interventionen als problematischer bzw. von sozialen Interventionen als unproblematischer einher. Der Fokus wurde so gewählt, weil die Arbeitsgruppe sich vor allem den ethischen Fragen zuwendet, die sich Ärzten und anderen im Gesundheitswesen tätigen Berufsgruppen stellen.

Insgesamt soll die Definition dazu dienen, das Arbeitsgebiet zu erfassen - sie ist nicht per se tauglich als moralisches Kriterium. Sie ist als deskriptives und analytisches, nicht aber als normatives Instrument zu verstehen. Für eine ethische Bewertung des heterogenen Feldes von Enhancementinterventionen müssen die moralisch relevanten Kriterien identifiziert werden, die sich unter anderem auf Aspekte wie Art der Intervention (Reversibilität, Risiko, Verschieben der individuellen Leistungsgrenze vs. der Speziesnorm usw.) und die Umstände der Anwendung (Urteilsfähigkeit des Betreffenden, Freiwilligkeit vs. sozialer Zwang) beziehen werden.

Dieses Verständnis von Enhancement ist in Einklang mit den Schlussfolgerungen der Expertengruppe «Zukunft Medizin Schweiz», die in ihrer Auseinandersetzung mit dem Krankheitsbegriff betont, dass nicht allein biologische Funktionsparameter, sondern auch subjektive, psychosoziale und kulturelle Faktoren zu berücksichtigen sind und die Übergänge zwischen Gesundheit und Krankheit Graubereiche bleiben [9]. Was wir als Krankheit verstehen, hängt - ähnlich wie beim Enhancement - nicht nur von den Funktionseinschränkungen und der subjektiven Befindlichkeit ab, sondern auch vom Umfeld, mit dem das betroffene Individuum interagiert, und von einem sozialen Aushandlungsprozess, wer die Leistungen, die Kranken gewährt werden (Zuwendung, Versicherungsleistungen usw.), für sich in Anspruch nehmen darf.

Wichtig für die Beschäftigung mit dem Thema «Enhancement» ist die Einsicht, dass der Krankheitsbegriff interessengeleiteten Ausweitungen bzw. Einschränkungen unterliegt. So hat zum Beispiel in den letzten Jahrzehnten im Zuge der Entwicklung von Psychopharmaka eine deutliche Ausweitung der psychiatrischen Diagnosen stattgefunden [10]. Kritiker verweisen auf eine zunehmende Medikalisierung z.B. von Befindlichkeiten oder Persönlichkeitsmerkmalen wie Schwermut oder Schüchternheit, mit der Konsequenz einer zunehmenden Standardisierung und dem Verlust persönlicher Identität und Authentizität [11]. Die Unterscheidung «medizinische Indikation - Therapie» und «keine Indikation vorliegend - Enhancement» mag zwar in formal-rechtlicher Hinsicht nützlich sein; die zugrunde liegenden ethischen, medizintheoretischen und gesundheitssoziologischen Fragen werden dadurch nicht gelöst.

Wenn es darum geht, den Begriff des Enhancements im weiteren Begriffsumfeld von Gesundheit und Krankheit zu situieren, so stellt sich auch die Frage nach der Beziehung zum Begriff der Prävention. Auch hier gibt es relevante Bezüge: Die Abgrenzung von Prävention und Enhancement ist ebenso unscharf wie die Grenzziehung zur Therapie: Sind 
synthetisch hergestellte Bakterien, die Arterien von Kalkeinlagerungen säubern, Prävention oder Enhancement? Diese Frage verliert allerdings an Relevanz, wenn man von der Zuteilung zur einen oder anderen Kategorie nicht erwartet, dass sie die normative Frage nach der moralischen Zulässigkeit löst. Eine Antwort auf die Frage, was Enhancement ist und was nicht, löst keineswegs die normative Frage nach der moralischen Zulässigkeit.

Ein weiterer Bezug ist hingegen von grosser Bedeutung: Wenn Enhancement als eine bisweilen medizinisch problematische gesellschaftliche Praxis verstanden wird, muss auch über eine Prävention von Enhancement - in gewisser Hinsicht vergleichbar dem Umgang mit Suchtmitteln - nachgedacht werden. Somit spielt Enhancement nicht nur mit Blick auf das Individuum und seine Präferenzen eine Rolle, sondern ist auch aus einer Public-Health-Perspektive zu betrachten.

\section{Fazit}

1. Enhancement - im Sinne der Verbesserung nichtpathologischer Merkmale - hat vielfach die Konnotation des Überflüssigen oder moralisch Fragwürdigen und ist doch zugleich ein Alltagsphänomen, in das auch verschiedene Bereiche der Medizin involviert sind. Was als Verbesserung verstanden wird, hängt dabei von den Erwartungen und Präferenzen der jeweiligen Gesellschaft ab.

2. Enhancementinterventionen sind vielgestaltig und unterscheiden sich in ethisch relevanter Hinsicht u.a. mit Blick auf die Invasivität der Eingriffe, die Irreversibilität sowie die Risiken für die individuelle und öffentliche Gesundheit. Die Zuordnung einer Intervention zu Enhancement löst somit nicht bereits die Frage nach der moralischen Zulässigkeit.

3. Enhancement wirft interessante rechtliche Fragen auf (etwa bezüglich der Einschränkung des Zugangs zu Substanzen oder bezüglich der Erstattungsfähigkeit von Kosten durch die obligatorische Krankenversicherung), doch ist der aktuelle gesetzliche Regelungsbedarf in der Schweiz umstritten.

4. Den Analysen und Empfehlungen zum Umgang mit Enhancement müssen eine Definition zugrunde liegen, damit der Rahmen abgesteckt und erkenntnistheoretische Prämissen offengelegt werden. Eine solche Definition muss der Relevanz des soziokulturellen Kontexts Rechnung tragen.

\section{Literatur}

1 Eckhardt A, Bachmann A, Marti M, Rütsche B, Telser H. Human Enhancement. TA-SWISS 56, vdf Hochschulverlag ETH Zürich; 2011. [1a] S. 14-18; [1b] S. 19-20; [1c] S. 191-234.

2 President's Council on Bioethics. Beyond Therapy: Biotechnology and the pursuit of happiness. New York: Dana Press; 2003.

3 Sauter A, Gerlinger K. Der pharmakologisch verbesserte Mensch: Pharmakologische Interventionen zur Leistungssteigerung als gesellschaftliche Herausforderung. Studien des Büros für Technikfolgen-Abschätzung beim Deutschen Bundestag, Bd. 34. Berlin: edition sigma; 2011.

4 Zonneveld L, Dijstelblowem H, Ringoir D (Hrsg.). Reshaping the Human Condition: Exploring human enhancement. The Hague: Rathenau Institute; 2008.

5 Science and Technology Options Assessment (STOA) European Parliament, Human Enhancement (IP/A/ STOA/FWC/2005-28/SC35, 41 \& 45). Brussels; 2009.

6 Sprumont D, Monbaron S-P. Le développement humain artificiel communément appelé «human enhancement»: Législation actuelle et besoins de réglementation, survol de la problématique. Etude juridique préliminaire, mandatée par la Commission nationale d'éthique pour la médecine humaine (NEK-CNE); 2010

7 Beck S. Die fehlende rechtliche Debatte einer gesellschaftlichen Entwicklung. MedR. 2006; 26(2): 95-102.

8 Juengst ET. Was bedeutet Enhancement. In: SchöneSeifert B, Talbot D (Hrsg.). Enhancement - die ethische Debatte. Paderborn: mentis; 2009: 25-45.

9 Schweizerische Akademie der Medizinischen Wissenschaften (SAMW) in Zusammenarbeit mit Experten und Expertinnen der Verbindung der Schweizer Ärztinnen und Ärzte (FMH) und der Medizinischen Fakultäten. Projekt «Zukunft Medizin Schweiz»: Ziele und Aufgaben der Medizin zu Beginn des 21. Jahrhunderts. Basel; 2004.

10 Angell M. The Epidemic of Mental Illness: Why? The New York Review of Books, June 23; 2011.

11 Elliott C. Better than well: American medicine meets the American dream. New York: Norton \& Company; 2003. 